\title{
Photorealistic VR Space Reproductions of Historical Kyoto Sites based on a Next-Generation 3D Game Engine
}

\author{
Wang Sheng ${ }^{1, *}, K^{2}$ Ishikawa ${ }^{1}$, Hiromi T. Tanaka ${ }^{2}$, Akihiro Tsukamoto ${ }^{3}$, \\ Satoshi Tanaka ${ }^{2}$ \\ ${ }^{1}$ Graduate School of Information Science and Engineering, Ritsumeikan University \\ ${ }^{2}$ College of Information Science and Engineering, Ritsumeikan University \\ ${ }^{3}$ The University of Tokushima \\ *cm013076@ed.ritsumei.ac.jp
}

Received: February 28, 2014; Accepted: November 14, 2014; Published: February 17, 2015

\begin{abstract}
Virtual reality (VR) technology can be used to simulate environments of historical sites in virtual spaces. In fact, many VR reproductions of historical sites have already been studied and developed. For users, the quality of the visual experience largely determines the authenticity of a VR space. However, when developing a VR space, it is difficult and timeconsuming to significantly improve visual quality while maintaining real-time rendering speed, which is the primary requirement of an effective user interface. For this reason, there have been few genuinely photorealistic VR spaces created for historical sites. However, this has begun to change due to the arrival of next-generation three-dimensional (3D) game engines. In this paper, we report on our recent development of photorealistic VR spaces for historical Kyoto sites created using the UDK 3D Game Engine. Specifically, we describe our VR reproduction of Reizei-ke, which consists of the Japanese court noble residence (kuge-yashiki), and Funehoko-cho, which is an important site for Japan's Gion Festival. Through the fabrication of these VR spaces, we could also extract a workflow that allows us to realize photorealistic and read-time VR contents of other cultural assets based on the abovementioned next-generation $3 \mathrm{D}$ game engine.
\end{abstract}

Keywords: Historical sites, VR technology, photorealistic rendering, next-generation 3D Game Engine, Digital Museum

\section{Introduction}

Historical sites, including the buildings and streets found at such locations, are carriers of a nation's cultural heritage and play an important role in helping us to understand life in bygone eras. To study the historical sites for their deep understanding or preserving them, 
real-time and high-quality environmental simulations are important. To realize such simulations, in recent years, computer graphics (CG) and virtual reality (VR) technologies have been widely used. And the simulations have contributed to management, reproduction, public exhibition, and scientific researches of the historical sites [1, 2, 3, 4].

S. Wells, B. Frischer, et al. created a virtual reconstruction of the ancient city of Rome that consisted of more than 7,000 three-dimensional (3D) models, which could be viewed on Google Earth. Furthermore, they also created a huge database related to the structures and tagged detailed information to each 3D model, so that users could view the information when they clicked the 3D models in the system [5, 6, 7]. Nobuaki Abe et al. imported high precision 3D shape models created with the help of a laser scanner into their VR content related to Maijishan Grotto in China [8]. Citizenship \& Technology at IBM provided a fully interactive virtual system of the Forbidden City in China that enabled role play and multi-user online accesses [9].

In general, to guarantee high interactivity, VR systems must realize real-time rendering. However, real-time rendering typically sacrifices visual quality because of its high computational costs, which are also a problem with highly detailed 3D CG models. Therefore, in most cases, visual quality must be compromised. Furthermore, even with the advanced VR spaces available today, high quality real-time rendering with highly detailed 3D shape models remains a challenge. On the other hand, the demands for high-quality visual contents, including photorealistic rendering, are becoming increasingly strident as display technology improves. However, in terms of VR content related to historical sites, only a few cases that realize both really photorealistic rendering and real-time rendering have been achieved [10, $11]$.

Amidst this situation, the release of DirectX 11 has brought the visual experience of 3D gaming fully into the next-generation. The relevant change is basically the improvement of the device rendering effects and realization of real-time global illumination. With this 3D game technology available, researchers began speculating on whether it could be applied to historical buildings. In their abovementioned study, E. F. Anderson et al. discussed the concept of "Serious Games" that play a role in the service of cultural heritage [12]. Furthermore, other researchers and groups have already attempted to utilize 3D Game technology into cultural heritage reproductions [13]. For example, Pudding Lane Productions, a team from De Montfort University, showed a particularly representative example involving the creation of a photo-realistically rendered VR reproduction of 17th Century London using a next-generation game engine [14].

This paper is the result of a research effort supported by the MR Digital Museum project sponsored by the Ministry of Education, Culture, Sports, Science and Technology (MEXT), Japan, and is devoted to building a Digital Museum capable of propagating a better understanding of Japanese art and culture to the world (see Fig. 1) [15]. Our team focuses primarily on the tangible cultural heritages in Kyoto, and has been working to create a photorealistically rendered VR reproduction of Kyoto's historical buildings in order to provide a high quality visual experience for users.

Simultaneously, high-detail 3D CG models and an advanced graphical user interface (GUI) system have been incorporated to facilitate strict comprehension and convenient interaction. We used a next-generation 3D game engine to realize the above goals. 


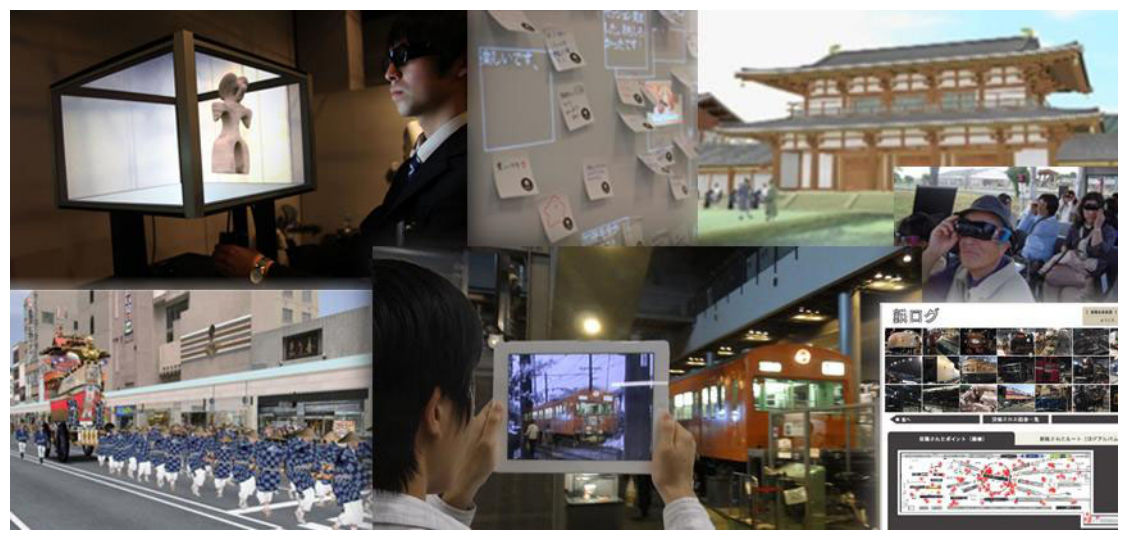

Figure 1: 2011 MR Digital Museum project (2010-2012, http://www.mr-museum.org).

The remainder of this paper is structured as follows. Section 2 provides an overview of the historical sites selected for inclusion in this paper. Section 3 explains the overall workflow involved with creating the VR spaces. Sections 4 and 5 present the results and system performance. Section 6 summarizes our achievement and describes possible future work.

\section{Historical spots to create their VR spaces}

In our previous work, we presented the entire city layout of Kyoto, as it existed during the Edo period, using an automatic urban space generation system that resulted in a 3D digital map [16]. In contrast, the work described in this paper involved allowing access to particular single structures or small-scale local constructions.

Herein, we describe the digital contents of two historical sites in Kyoto. The first is the oldest existing Japanese court noble residence (kuge-yahiki) in Kyoto, which is known as the Reizei-ke residence. This is significant because court noble residences are one of the most culturally important architectural types found in old eras of Kyoto. The second is the Funehoko-cho area, which is the starting point and goal of activities during the Gion Festival, which is one of Japan's three major festivals.

\subsection{Reizei-ke residence}

The history of the Reizei family can be traced back to the Heian period of Japan (704-1192 AD). Nagaie, the sixth son of Michinaga Fujiwara, established the Mikohidari-ke (Mikohidari Family). After about 200 years, Tamesuke, one of Nagaie's descendants, renamed the family, which then became known as the Reizei-ke. Nevertheless, since the days of Nagaie, the family has specialized in "waka", which is a three-one Japanese poetry form. Thus, the Reizei-ke has been considered a leading family in the history of Japanese poetry.

In modern times, the family established the Reizei-ke Shiguretei archive, in which over a thousand books, paintings, and calligraphy artworks have been preserved. The Reizei-ke 

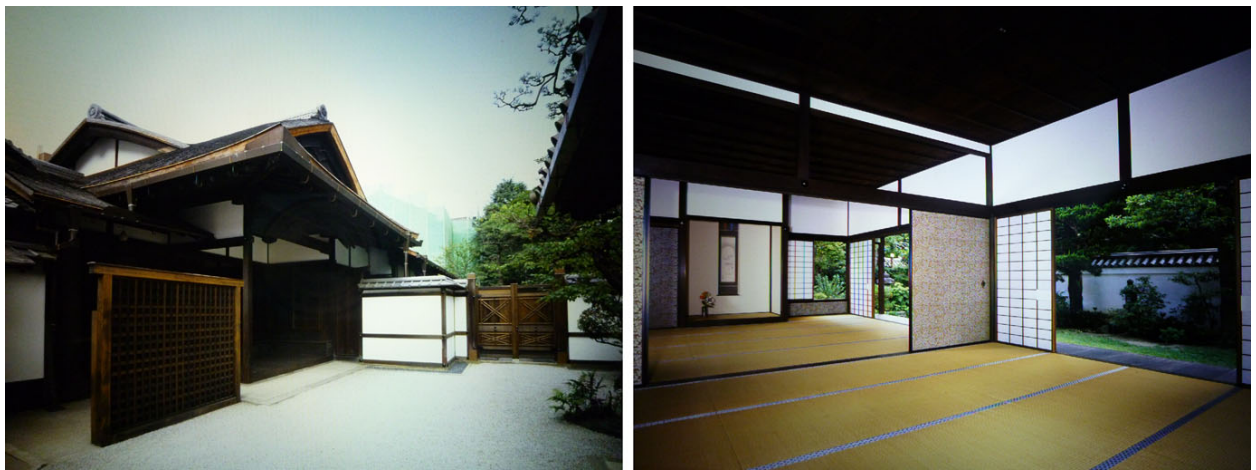

Figure 2: Photographs of Reizei-ke residence. The left side shows the grand entrance, while the right side shows the corner of a tatami mat room.

residence is located in the north of the Kyoto Imperial Palace, and is the only court noble residence that still exists in Kyoto City. In 1979, the Reizei-ke residence and the Reizei-ke Shiguretei archive were designated as important cultural properties of Japan. Currently, the residence is well preserved, but remains closed to the public for most of the year. Fig. 2 shows the exterior and interior views of the Reizei-ke residence.

\subsection{Funehoko-cho}

One of the highlights of the Gion Festival is the Yamahoko parade. One of the most famous floats displayed during the parade is the Funehoko float, which is celebrated for its unique boat-like shape and gorgeous tapestries. The Funehoko float is one of the most significant cultural assets in our Digital Museum project. Funehoko-cho is the name of the street area where the local residents maintain the Funehoko float. The Funehoko-chokaisho, which is a trysting house maintained by the Funehoko Preservation Society, and is both the starting point and the goal of the Fuhehoko float during the Yamahoko parade. A number of events are held in the Funehoko-chokaisho during the Gion festival. Fig. 3 shows photos of the Funehoko and the Funehoko-chokaisho.

\section{The Workflow of VR Space Creation}

We used the next-generation Unreal Development Kits (UDK) 3D game engine [17], which supports photorealistic and real-time rendering, to construct the Reizeike residence and Funehoko-cho VR spaces. UDK is a functionally limited free version of Unreal Engine 3 , that retains most of the powerful rendering techniques of the full version. This makes it possible to achieve photorealistic rendering. In contrast, Unity 3D is also famous for its powerful tools and convenient usage. However, UDK has better rendering performance that fits the purpose of our work. On the other hand, the 3D game engines used for general commercial purpose (e.g. Unreal Engine, Cryengine, Frostbite) are not in the range of options at present. 


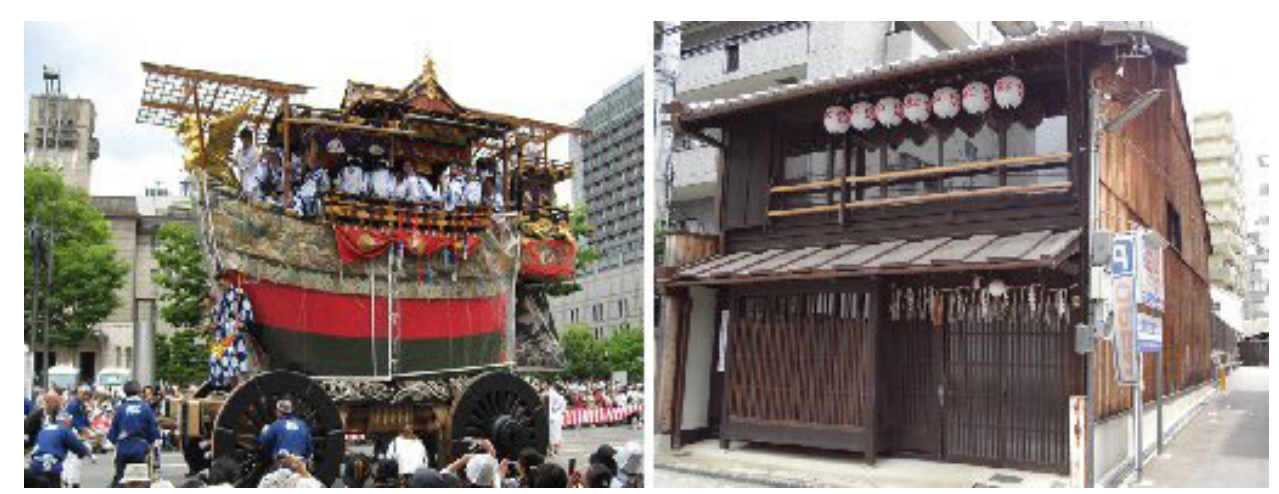

Figure 3: Photographs of Funehoko (left) and Funehoko-chokaisho (right).

When constructing these VR spaces, we also extracted a workflow that can be used to realize photorealistic and read-time VR contents of other cultural assets based on nextgeneration 3D game engines. The entire workflow is divided into two parts. The first part is the creation of 3D shape models while the second part is the construction of the VR spaces themselves. The following subsections describe the two parts in detail. The pipeline of the workflow is shown in Fig. 4. The difference between our workflow and those of existing approaches is that we incorporate multi-layered material creation and global illumination, which have become efficiently implemented in the recent 3D games powered by the next-generation game engine, but have seldom been mentioned in common VR spaces of historical sites.

\subsection{D Shape Model Creation}

The 3D shape models, such as houses placed in our VR space, are first modeled in an optimal resolving power (We set it to $10 \mathrm{~cm}$. In UDK, a higher resolution 3D shape model may cause unwanted artifacts). Meanwhile, the boundary-shape information required for collision detection was also created in this phase. To present the detailed patterns that were not included in the 3D shape models created with the current resolving power, we applied bump maps, normal maps, and specular maps on the basis of texture mapping. Photographs of the actual objects were hereby used rather than general template textures to ensure the authenticity. We used Autodesk Maya and Adobe Photoshop to handle these work. However, the diffuse attribute (color information) was only applied to the 3D shape models at this time. The bump, specularity, and transparency are reset in UDK at next phase, because these properties are lost when the models are exported to UDK. In addition, 2 UV channels, both of which present the surface of a 3D shape model by a 2D image, were assigned to each 3D shape model. The first UV channel is for texture mapping as general usage, while the second UV channel is used for storing global illumination data, so called light map, which is generated after the whole scene has been set up in UDK. Finally, the 3D shape models were exported to UDK through intermediate FBX (Filmbox) format files. 


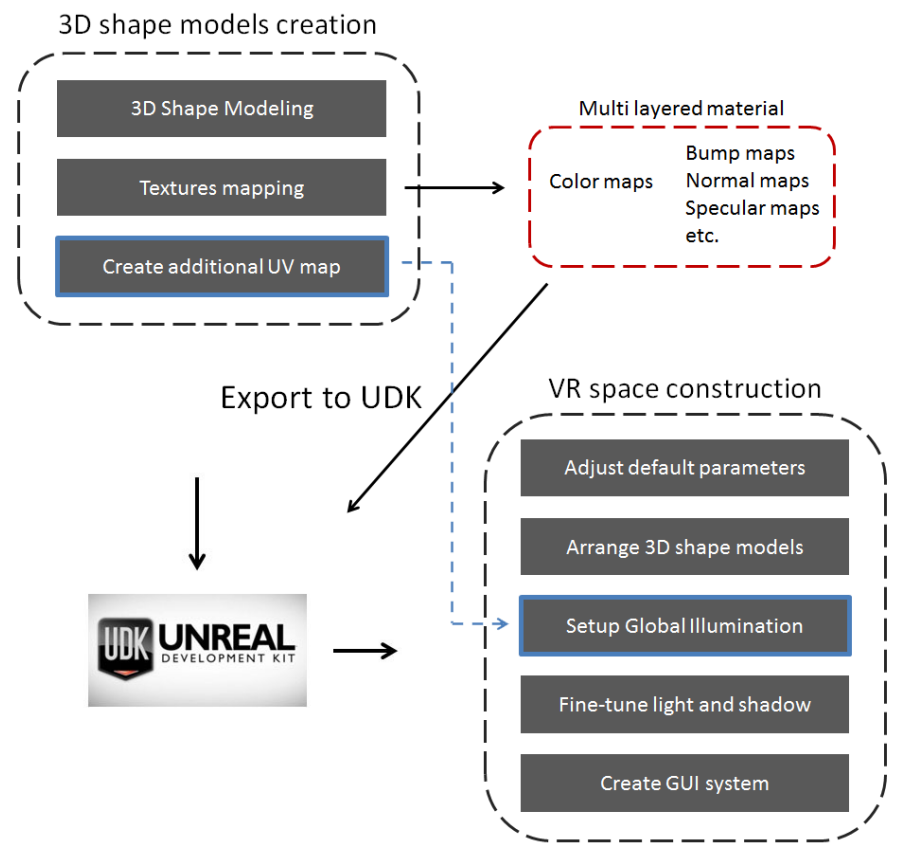

Figure 4: Schematic illustration of the workflow. The difference between our workflow and those of existing VR space workflows is that we incorporate multi-layered material and global illumination. The red dotted line in the figure shows that the multi-layered material data is created at the phase of 3D model creation, and linked to the corresponding 3D shape model afterwards by UDK. The sub-steps highlighted by blue lines show that the global illumination is achieved on the basis of the second UV map created at the first part of the workflow. 


\subsection{VR Space Construction}

UDK provides a sample stage in which the basic VR elements such as terrain, sky-dome, and the character movement function, have already been implemented. Accordingly, we constructed our VR space based on the sample stage. The work in this part was done following the five steps below:

(1) The default settings of the sample stage, for example, the color of light, character movement, and the speed/action, are first adjusted to suit our VR space instead of a fast-paced first person shooting game. Since our VR space is intended for use in a digital museum, the 3D scenes are designed to be comprehensible rather than stimulable.

(2) The 3D models created at previous phase are then placed in their correct positions in relation to the real world and additional designs, if any. Meanwhile, the data related to surface bump, normal, specularity, and transparency are added to generate a multilayered material file for each linked 3D model. These kinds of material data are created manually so that they may need to be fine-tuned several times until the result is acceptable.

(3) To implement global illumination, a light map is pre-calculated based on the rule of global illumination for each 3D shape model. The result is then saved to the corresponding 3D model's second UV channel. This step also needs to be retuned because artifacts might occur for an improperly created UV map (the second one) or inadequate precision of light map generation.

(4) The rendering expresstion is optimized afterward for unnatural shading presentation. Additional light sources may need to be implemented when there has been a complex light environment (e.g. scenes that contain both lights from outdoor and indoor).

(5) Finally, Adobe Flash Pro was used to design and implement a GUI to facilitate convenient user interaction. The result is output in SWF file format so that it can be managed by UDK.

\section{VR Space of the Reizei-ke Residence}

Court noble residences are one of the most culturally important architectural types representative of Kyoto's previous eras. Therefore, the ability to allow users to observe the architectural structure of the Reizei-ke in VR space is quite significant. To show the most detail as possible, a 3D shape model of the Reizei-ke residence was created in precise accordance with the construction report of repair work performed from 1994 to 2000. Fig. 5 shows a perspective image of the resulting 3D shape model.

Since the construction report describes only the tatami mat rooms and the kitchen, we modeled the outer wall and the garden based on photographs and a design drawing. Moreover, a number of the residences' 3D decorations were modeled to enrich the overall VR 


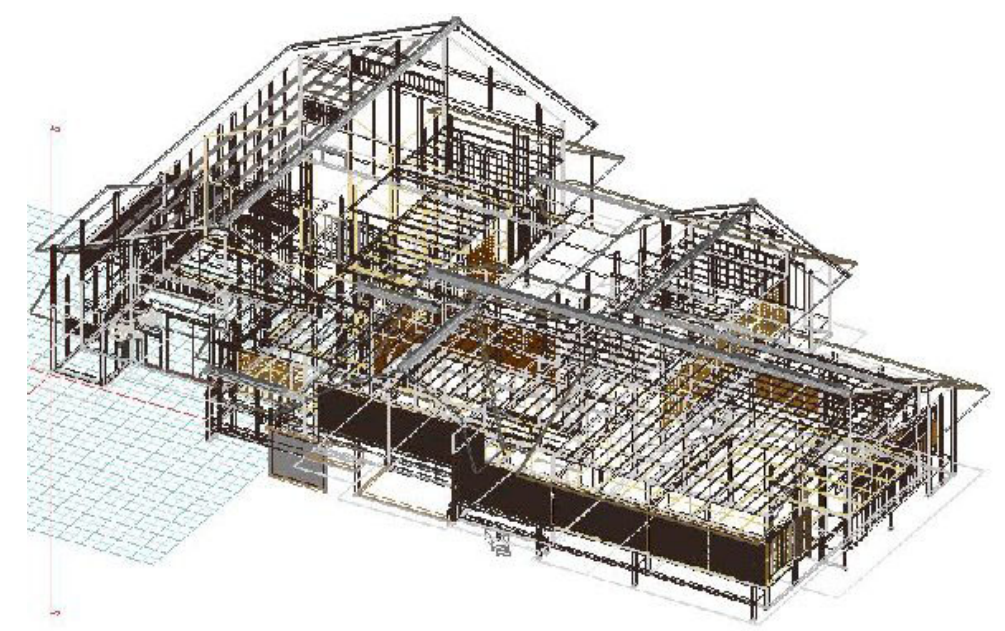

Figure 5: Perspective image of the high-detailed 3D shape model of the Reizei-ke residence.

space experience. Fig. 6 shows the design drawing we referred to during the modeling process. As for texture mapping, textures were extracted from photographs acquired during the opening of the Reizei-ke residence to the public in 2009 and 2011. Fig. 7 shows an example of texture extraction from a photograph. Besides, the bump, normal, and specular maps were created afterward and were arranged to multi-layered material data used to present advanced expression of object surfaces. Fig. 8 shows the components of a material file of fusuma (a sliding door used to partition off rooms in a Japanese house).

As previously mentioned, the VR space of Reizei-ke was created using the sample stage of the UDK software package. However, since the sample stage of the product was designed primarily to provide stimulable effects suitable for gaming, it was first necessary to modify or delete some of the default settings in order to produce a comprehensible VR space more suitable for experiencing a cultural asset. For example, the moving speed of the character was reset to a slow pace to ensure that users feel themselves as visitors rather than fighters or soldiers.

Formal gardens are one of the most important features of a Japanese court noble residence. Therefore, to a significant extent, the expression of the garden reflects on the look and feel of the VR space. To ensure a high level of realism, the texturing of the garden had to be carefully orchestrated. Multi-layered textures were used to achieve a smooth and natural expression of gravel and vegetation, and the terrain was rebuilt to form the pond, the water of which was presented with an animated texture.

The most difficult part of the rendering was proper expression of the plant life. Fortunately, UDK provides a vegetation-modeling tool called SpeedTree, which enables fast plant modeling, and which also supports the animation of the wind effect. This tool partly simplified the creation of plants. Fig. 9 shows the final rendering result of the garden.

Another important component that affects the rendering quality is the expression of global illumination. To enable global illumination with UDK, each 3D shape model must 


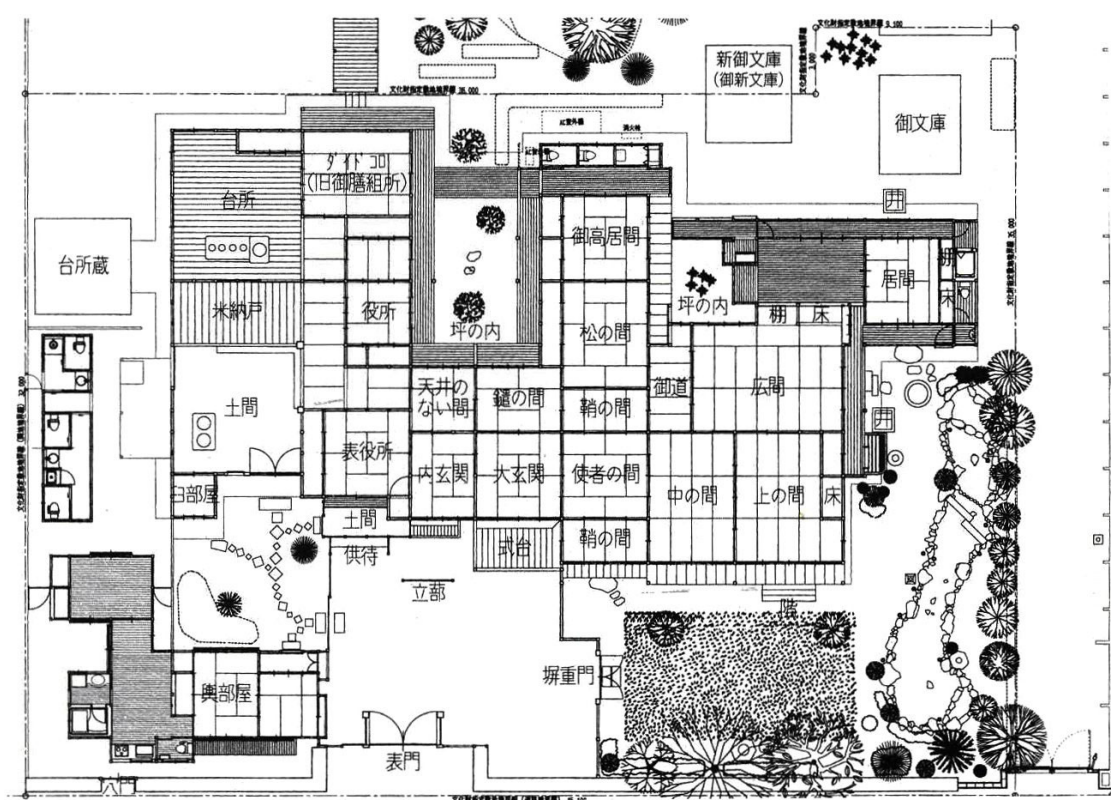

Figure 6: Design drawing of Reizei-ke residence.
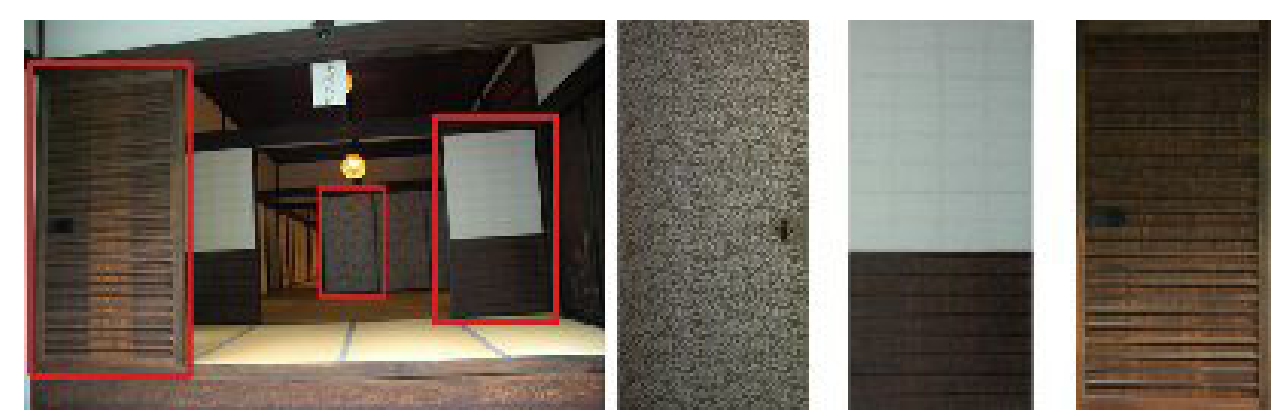

Figure 7: Example of texture extraction from a photograph. 


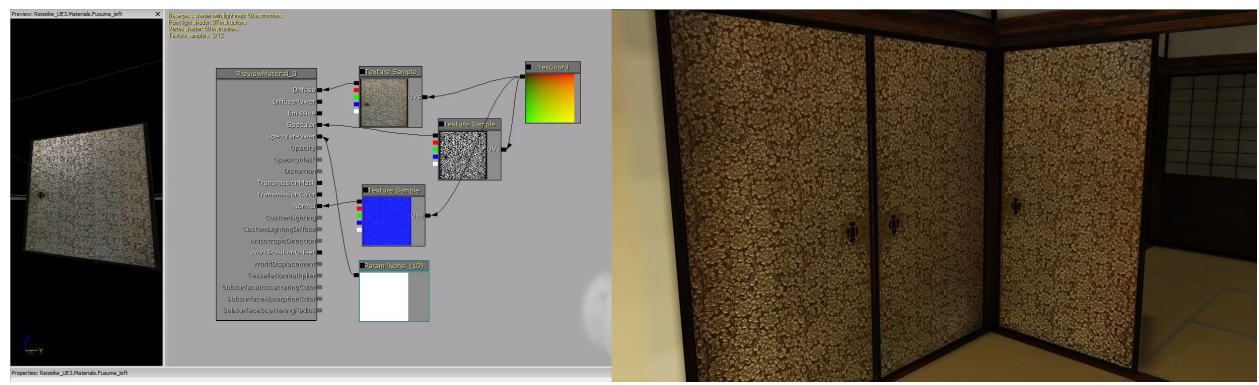

Figure 8: Karakami (a kind of high-class patterned paper) is mainly used to ornament fusuma in Reizei-ke residence. It presents an unique visual effect due to its uneven surface and special material. A normal map and a specular map were added to the material setting to express the advanced visual effect. (Left) The material setting of fusuma in UDK. (Right) A rendering result of fusuma in Reizei-ke Residence.

have two UV channels, one is for texture mapping while the other is for saving a light map that describes the lighting details on the 3D model surface. Fig. 10 shows the rendering results of a tatami mat room with and without global illumination. As can be seen in the figure, the use of global illumination is very effective for improving the look and feel in the VR space.

The average rendering speed is 30 frames per second (FPS) for a $1920 \times 1080$ screen image. The total data size of the VR space is 320 megabytes. The hardware environment is summarized in Table 1. These experimental results show that the real-time rendering is successfully achieved for a high-resolution image size.

Table 1: The runtime environment of our VR space

\begin{tabular}{ll}
\hline Item & Value \\
\hline Operating System & Windows 7 64bit \\
CPU & Intel(R) Core(TM) i7-2600 3.4 GHz \\
Memory & 12 GB GDDR3 \\
Graphic Card & AMD Radeon HD 6670 \\
Video Memory & 1024 MB GDDR5 \\
\hline
\end{tabular}

Fig. 11 (left) shows a photograph of the Reizeike-residence. On the other hand, Fig. 11 (right) shows the modeled Reizeike-residence in our VR space. In the VR space we can flexibly tune, for example, seasons. Fig. 11 (right) is created by setting the environmental parameters to summer. Such environmental simulation is executable easily and realistically.

To facilitate digital museum usage, a start page containing the history, stories, photographs and other information of Reizei-ke is incorporated into the VR space, and a widget that shows the current location of the viewer was created (see Fig. 12). 

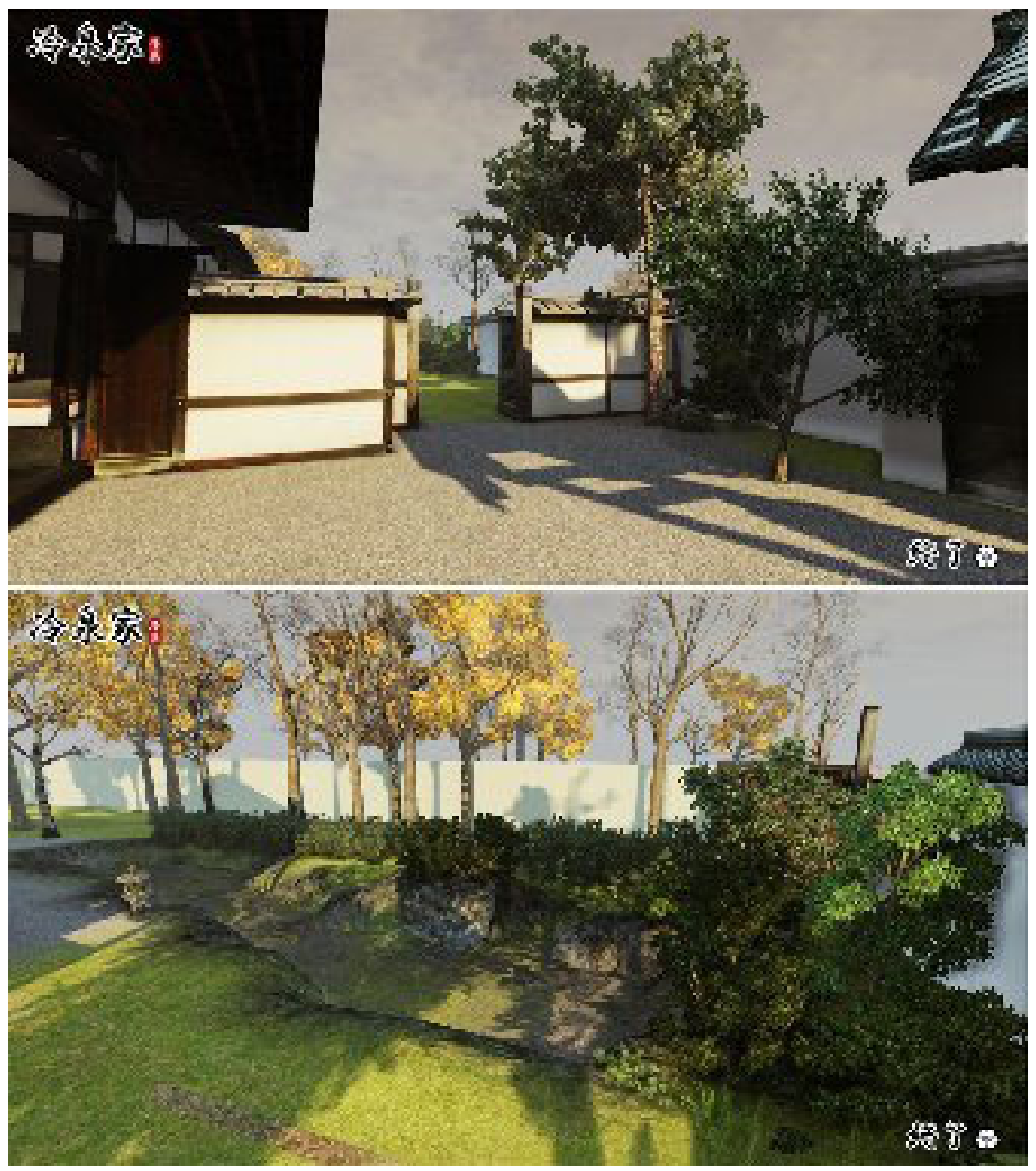

Figure 9: Snapshots of the garden in our VR space of Reizei-ke. 

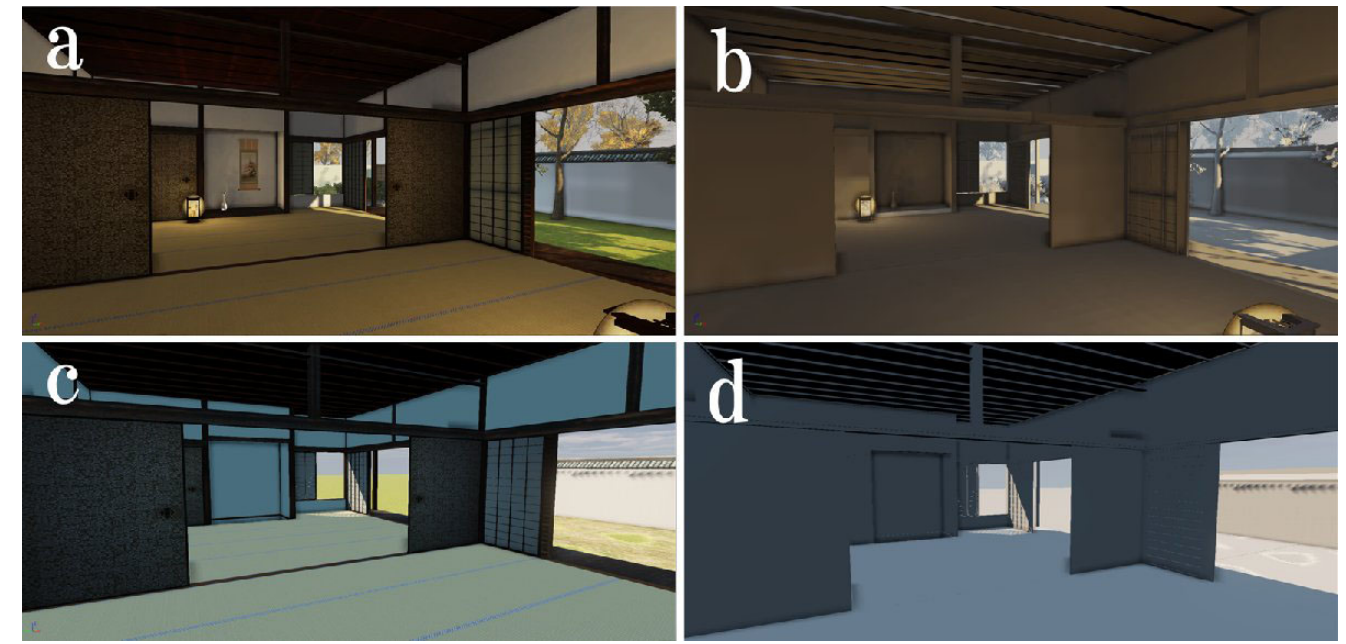

Figure 10: (a) Snapshot of our VR space of Reizei-ke residence with global illumination activated. (b) Image to show raw illumination effects without texture mapping for (a). (c) Rendering result without global illumination. (d) Image to show raw illumination effects without texture mapping for (c).
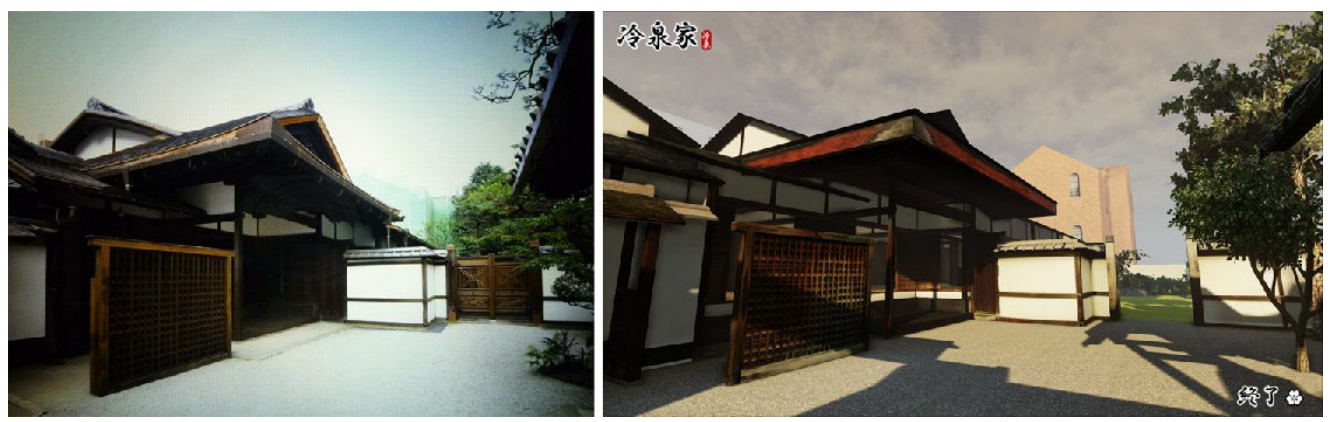

Figure 11: Comparison of visual experience. (Left) shows a photograph of grand entrance of Reizei-ke residence. (Right) shows the final rendering result of the VR space of Reizei-ke. 

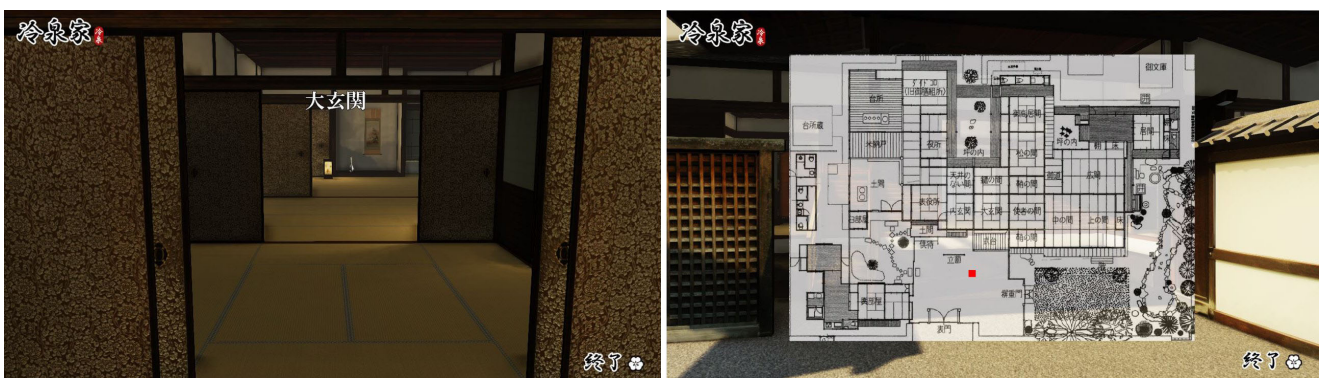

Figure 12: (Left) The prompter that shows the name of the current place. (Right) A map can be invoked interactively, in which a red point shows the current position of the user.

\section{VR space of Funehoko-cho}

Rather than a single residence, Funehoko-cho is the Kyoto district where the Gion Festival takes place every year. This is a culturally important event in Japan. The Funehoko-cho VR space is uniquely suitable for use as a platform for a virtual rendering of the Gion Festival because it is both the starting point and the goal of the Funehoko float in the Yamahoko parade. Therefore, a good expression of the Yamahoko parade in a VR space could be expected to provide suitable contents for a digital museum.

Furthermore, the Funehoko-cho area includes a number of famous Machiya (traditional townhouse) such as the Funehoko-chokaisho trysting house and Nagae-ke house. Since, traditionally, many commoners in the Kyoto urban area have lived in such Machiya, these structures are also culturally important architecture types in Kyoto.

Using the workflow described in Section 3, we created high-precision VR spaces of Funohoko-cho street area as well as Funehoko-chokaisho as they existed in the Edo era. To accomplish this, a number of Machiya were automatically created using software developed in our previous work [16].

Unlike the Reizei-ke VR space, the history and detailed information of each structure were integrated into the VR space using pop-up widgets rather than as a start page, a feature that allows users to obtain descriptions directly while "walking" through the VR space. Fig. 13 shows snapshots of the Funehoko-cho VR space in an old-time era of Japan (Edo Period). Unlike the Reizei-ke VR space, the history and detailed information of each structure were integrated into the VR space using pop-up widgets rather than as a start page, a feature that allows users to obtain descriptions directly while "walking" through the VR space. These widgets can be regarded as interfaces of interactive environmental simulation of Funeboko-cho during an old days of Japan.

We also evaluated rendering speeds of virtual walk-through in our VR space of the Funehoko-cho. The total data size of the VR space is 50 megabytes. The Funehoko-cho VR space operates under the same VR space environment as Reizei-ke. But the FPS of the Funehoko-cho was better because it could be stabilized at 60 FPS due to the smaller number of plants. 

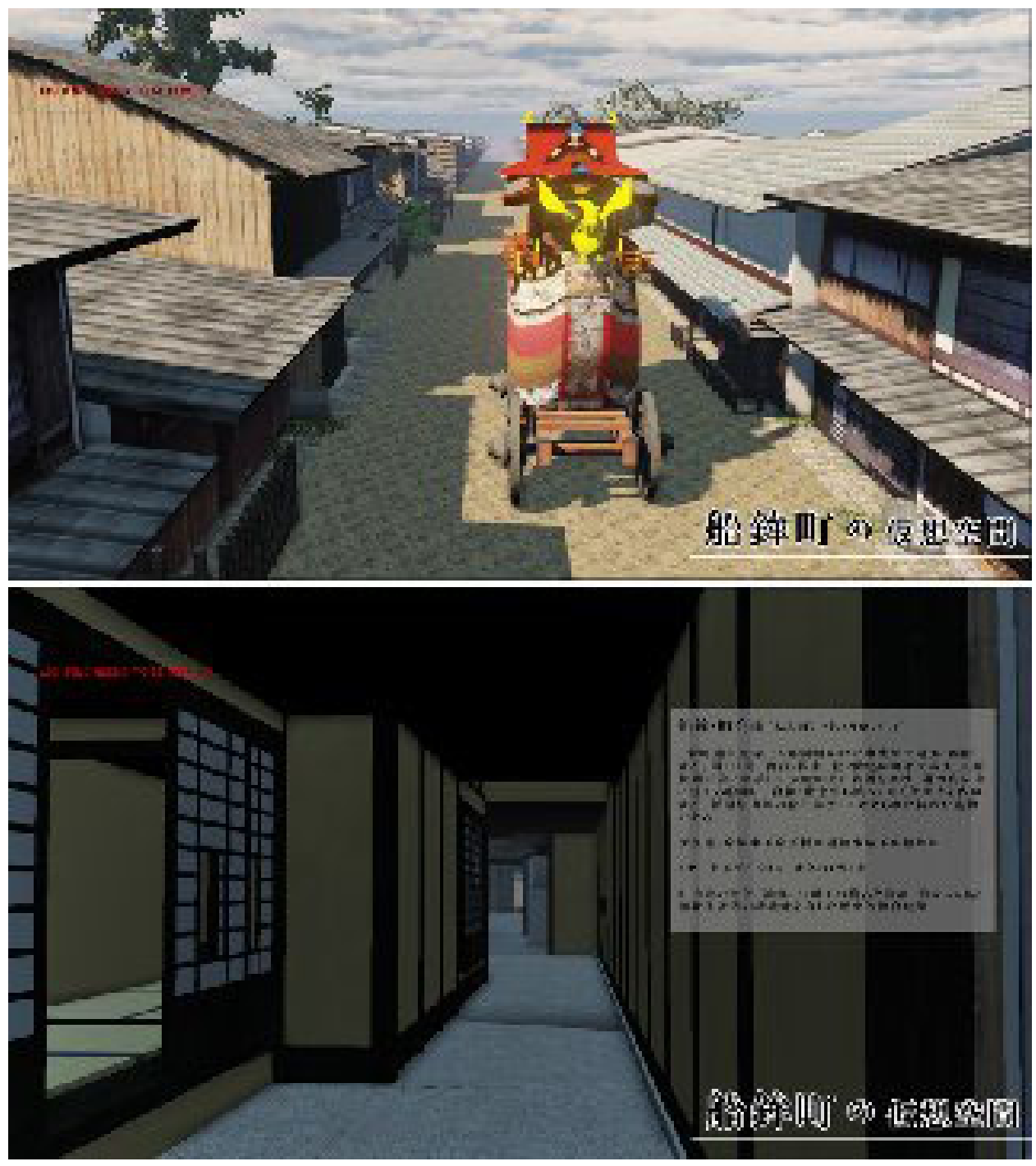

Figure 13: Snapshots of the VR space of Funehoko-cho. (Top) shows the center of Funehoko-cho. (Bottom) shows the interior of Funehoko-chokaisho. An information box is shown in the right side of the picture. 


\section{Conclusions and Future Work}

We reported our recent development of photorealistic VR spaces of historical sites in Kyoto to be used for real-time and high-quality environmental simulation. By using one of the next-generation 3D game engines, we created the VR space of Reizei-ke, which is the oldest existing Japanese court noble residence (kuge-yashiki), and the VR space of Funehoko-cho, which is an important spot for the Gion Festival in Japan. Our experiments using these VR spaces show that over 30 FPS can be realized for the practical 3D models of cultural assets. The rendering quality is photorealistic enough incorporating effects of multi-layered material and global illumination. Through constructing these VR spaces, we could also extract a reliable workflow to create real-time rendered and photorealistic VR spaces of cultural assets based on a next-generation 3D game engine.

The two VR spaces were made public for over 1,500 people at the exhibition of "Digital Museum of Gion festival 2014" held at The Museum of Kyoto, July 22-25, 2014. Through reviewing the feedbacks from the visitors, our productions were highly rated within all presentations. Some visitors who have acquaintance with the Reizei-ke and Funehoko highly affirmed the authenticity and the rendering experience. On the other hand, the children enjoyed exploring the scenes, which reflected the advantage of high interactivity of the VR spaces. We also received many advices from the visitors, such as the incorporation of the augmented reality, which would be the basis of our future work.

In this paper, we mainly focused on rendering quality and rendering speed of VR spaces. To utilize the VR spaces as contents of digital museums, we need to consider proper user interfaces and output devices, too. Fortunately, recent 3D game engines support excellent interactive user interfaces such as various types of game controllers. Besides, the nextgeneration 3D game engines support various output devices such as tablet-type devices and head-mounted displays (HMD). As long as we use the next-generation 3D game engine, use of these modern user interfaces and output devices are quite easy, which should lead to new advantages of digital museums.

In future, we plan to incorporate laser-scanned real 3D cultural objects into our modeled VR spaces. Such fusion of modeled and scanned (measured) objects should further enhance the possibility of digital museums.

\section{Acknowledgement}

We would like to thank the members of the Kyoto Heritage Preservation Association and Prof. Tamehito Reizei for their cooperation during this project. The Photographs of Reizei ke residence (Fig. 2 and Fig. 11) are used with permission by Reizei-ke Shiguretei archives. We would also like to express our gratitude to the Funehoko Preservation Association and Prof Susumu Nakata for their advice. This study is funded by the "Research and Development of Demonstration Experiment System for the Advancement of Digital Museums" research project sponsored by the Ministry of Education, Culture, Sports, Science and Technology (MEXT), Japan. 
Journal of Advanced Simulation in Science and Engineering

\section{References}

[1] E. Berndt, J. Carlos: Cultural heritage in the mature era of computer graphics, Computer Graphics and Applications, IEEE, 20:1 (2000), 36-37.

[2] A. C. Addison: Emerging trends in virtual heritage, MultiMedia, IEEE, 7:2 (2000), $22-55$.

[3] R. Stone, T. Ojika: Virtual heritage: what next?, MultiMedia, IEEE, 7:2 (2000), 73-74.

[4] A. Gaitatzes, D. Christopoulos, M. Roussou: Reviving the past: Cultural Heritage meets Virtual Reality, Proceedings of the 2001 conference on Virtual reality, archeology, and cultural heritage, New York, 2001, 103-110.

[5] The Rome Reborn project http:// romereborn.frischerconsulting.com/.

[6] S. Wells, B. Frischer, D. Ross, C. Keller: Rome reborn in Google Earth, Computer Applications and Quantitative Methods in Archaeology, Williamsburg, 2009, 365-371.

[7] K. Dylla, B. Frischer, P. Mueller, A. Ulmer and S. Haegler: Rome reborn 2.0: a case study of virtual city reconstruction using procedural modeling techniques, Computer Applications and Quantitative Methods in Archaeology, Williamsburg, 2009, 62-66.

[8] N. Abe, T. Kawai, J. Ohya, H. Zha, M. Ando: Digital Archiving of Maijishan Grotto and Stereoscopic VR Content, IEEE Computer Graphics and Applications, 14:3 (2009), 275-282.

[9] The Forbidden City: Beyond Space \& Time:

http:// www-03.ibm.com/press/us/en/pressrelease/25379.wss/.

[10] S. Maass, M. Trapp, J. E. Kyprianidis et al. : Techniques for the interactive exploration of high-detail 3D building reconstructions using the example of Roman Cologne, Computer Applications and Quantitative Methods in Archaeology, Budapest, 2008, 223229.

[11] G. Papagiannakis, G. L' Hoste, A. Foni, N. M. Thalmann: Real-time photo realistic simulation of complex heritage edifices, Proceedings of Seventh International Conference on Virtual Systems and Multimedia, Berkeley, 2001, 218-227.

[12] E. F. Anderson, L. McLoughlin, F. Liarokapis, C. Peters, P. Petridis, S. D. Freitas: Serious Games in Cultural Heritage, 10th International Symposium on Virtual Reality, Archaeology and Cultural Heritage, Valletta, 2009, 29-48.

[13] A. Merlo, L. Dalco, F. Fantini: Game engine for Cultural Heritage: New opportunities in the relation between simplified models and database, 18th International Conference on Virtual Systems and Multimedia, Milan, 2012, 623-628. 
[14] Pudding Lane Productions:

http:// puddinglanedmuga.blogspot.jp/.

[15] MR Digital Museum Project 2011:

http:// www.mr-museum.org/.

[16] M. Sawai, Y. Isoda, A. Tsukamoto, Y. Kosaka, K. hasegawa, S. Nakata, S. Tanaka: Automatic Modeling of Virtual 3D Streets Based on GIS Data: Application to Generation of Kyoto in the Edo Era, Proceedings of 5th International Conference on Intelligent Information Hiding and Multimedia Signal Processing, Kyoto, 2009, 1138-1143.

[17] A. Thorn: UDK Game Development, Course Technology Inc. Publication, 2011. 